\title{
Call Auctions with Contingent Orders
}

\author{
Isa E. Hafalir* and Serkan Imisiker ${ }^{\dagger \ddagger}$
}

November 8, 2011

\begin{abstract}
We introduce a new mechanism for call auctions which are widely used in stock exchanges. By incorporating contingent claims (buy stock A, if selling stock B) into the price discovery process, we achieve a higher liquidity in call auctions and lower volatility after opening call auctions. We also show that present call auctions and the proposed mechanism have similar incentive properties.
\end{abstract}

Keywords: Call Auctions, Stock Exchanges, Volatility

JEL Codes: G20, D44

\section{Introduction}

Intraday price volatility of stocks generally tends to be higher at the opening interval than other trading time periods during the day. Among others, Lockwood and Linn (1990) support this claim using the data of Dow Jones Industrial Average between 1964-1989. Most stock exchanges around the globe implement call auctions at the opening and closing of trading sessions in order to make an effective system for price discovery, reduce the opening volatility, and avoid closing price manipulations.

In this manuscript, we introduce a new call auction mechanism with an innovative algorithm. This new design lets prospective investors to place contingent buy orders and determines the auction price by using these contingent orders, which will help to

*Tepper School of Business, Carnegie Mellon University, 5000 Forbes Ave, Pittsburgh, PA, USA. Email: isaemin@cmu.edu.

${ }^{\dagger}$ Corresponding Author. Tepper School of Business, Carnegie Mellon University, 5000 Forbes Ave, Pittsburgh, PA, USA and Capital Markets Board of Turkey, Ankara, Eskisehir Yolu, No: 156, Turkey. Phone: +1 412 5194616. Fax: +1 412 268-7930. Email: serkani@andrew.cmu.edu. Permenant Address: Capital Markets Board of Turkey, Ankara, Eskisehir Yolu, No: 156, Turkey.

${ }^{\ddagger}$ We would like thank Tevfik Kinik, Duane Seppi, and Chester Spatt for useful discussions. Isa Hafalir thanks National Science Foundation for grant SES 0752931. All views expressed herein are solely those of the authors, and do not necessarily reflect those of the Capital Markets Board of Turkey. 
solve the problems mentioned above. Our mechanism is also theoretically shown to increase the liquidity level in auctions. We also consider the incentive properties of present call auctions and the proposed new mechanism and find that they are similar.

Investors regularly update their portfolio decisions with incoming news received overnight, and they may need to change their holdings at the forthcoming trading day. But if they want to realize this change at the opening session in order to benefit from the concentration of liquidity at the call auction, in some cases these traders may not have sufficient sources to execute their buy orders before completing their sell orders. One can argue that the actual delivery and payment (settlement) procedure is not instantaneous in many stock exchanges; therefore traders can do this exchange without using contingent orders. However, financial intermediaries are exposed to settlement risk, and the risk management considerations increase the transaction costs of these traders. On the other hand, contingent orders reduce transaction costs and provide the opportunity of simultaneously buying and selling different stocks at the opening or closing call auction.

Other potential groups of investors who may use contingent orders are "pairs traders." Pairs trading is a well-known and commonly used trading strategy, which is formed by, at first, finding two stocks that are highly correlated with each other depending on historical records, then, buying one and selling the other one whenever the spread between them increases significantly. Gatev et al. (2006) show empirically that this simple strategy brings up to $11 \%$ average annual excess return. They also note that the profitability of this strategy, used largely by institutional investors, depends on the traders' transaction costs. It has also been shown that call auctions are preferable to continuous auctions for these investors, as call auctions exhibit lower transaction costs and less market impact (see Snell and Tonks 2003 and Economides and Schwartz 1995). Also, the reference price property of closing auction prices attracts institutional investors to be more active and aggressive at the closing auction (Pagano and Schwartz 2003). An arbitrageur, with a pairs trading strategy, can avoid the risk of "buying a lower priced stock without selling a higher priced one" by using contingent orders in our proposed auction market.

\section{Call Auctions}

Different stock exchanges use very similar types of uniform price auction mechanism for their call auction sessions. We demonstrate the general idea of these auctions below.

\subsection{Current Call Auction Mechanism}

Investors can only enter the call auction with limit orders, which consist of the code

of the individual stock, price, and quantity for the order. In the call auction $(C A)$ 
buyers and sellers announce their values and quantities $(v, q)$. Then the call auction price $(C A P)$ is chosen to be one of the prices that maximize the total sales (total sale with a price $p$ is defined as the minimum of "total quantities demanded by buyers with values $v \geq p^{\prime}$ and "total quantities supplied by sellers with values $v \leq p$ "). In case there are multiple maximizers of the total sale, the price closest to the last closing/sale price is chosen. For CAP equal to $p$, there could be excess demand or supply. In case of an excess demand (supply), then all matching sell (buy) orders will be executed, and the lowest buy (highest sell) offers may be partially executed. In case of multiplicity of lowest buy (highest sell) offers, these offers will be executed on a pro rata basis.

\subsection{Call Auction Mechanism with Contingent Orders}

Consider an investor who holds stock A and would like to buy stock B only if she sells stock A. Now, suppose we allow investors to announce their orders as "I would like to buy Stock B (at most price $x$ ), if I can sell Stock A ( $q$ units of the stock for a price at least $y) . "$

After all regular limit orders and contingent orders are collected until the call auction, the Call Auction with Contingent Orders (CACO) mechanism is run by the following algorithm in order to determine the auction prices (CACOP) of different stocks:

Stage 1: All regular limit orders and selling limit orders of the contingent orders will be entered and the price vector $p_{1}$ will be determined according to CA mechanism described above. If any of the contingent selling limit orders is executed with $p_{1}$, corresponding buying limit orders of these contingent orders ${ }^{1}$ are entered into the auction book and the algorithm moves to Stage 2 .

Stage k: CA mechanism determines the new price vector $p_{k}$ with the updated orders in the auction book. If any of the contingent selling limit orders is executed with $p_{k}$, corresponding buying limit orders of these contingent orders are entered into the auction book and the algorithm moves to Stage $k+1$. Otherwise the algorithm ends with the resulting CACOP vector $p_{k}$.

One very important feature of this mechanism is that at each stage $k$, only new buy orders can be added to the auction book. Hence, matched sellers of stages $1, \ldots, k-1$ will also be matched in stage $\mathrm{k}$ and $p_{k}$ is nondecreasing in $k$. This feature guarantees that there will be no loops in the algorithm and it will end in finitely many steps. We now illustrate our mechanism with an example.

\footnotetext{
${ }^{1}$ The quantity of the contingent buy order is calculated by "current revenue of the investor at that stage" over "buying price announced in the contingent order, $x . "$
} 


\subsection{Example:}

Consider the following opening order books with two stocks.

Stock A's Limit Order Book

(Last Closing Price is 1.00)

Buy Orders Sell Orders

\begin{tabular}{rr|rrl}
\hline \hline Price & Quantity & Quantity & Price & \\
\hline 1.01 & 10,000 & 7,000 & .98 & \\
1.00 & 5,000 & 12,000 & .99 & $C(.52)$ \\
.99 & 15,000 & 4,000 & 1.00 & \\
.98 & 7,000 & 6,000 & 1.01 & \\
.97 & 8,000 & 8,000 & 1.02 & \\
& & 4,000 & 1.03 &
\end{tabular}

Table 1
Stock B's Limit Order Book

(Last Closing Price is .52)

\begin{tabular}{rr|rrr}
\multicolumn{2}{c}{ Buy Orders } & \multicolumn{3}{c}{ Sell Orders } \\
\hline \hline Price & Quantity & Quantity & Price & \\
\hline .51 & 8,000 & 12,000 & .50 & \\
.50 & 6,000 & 9,000 & .51 & $C(1.01)$ \\
.49 & 12,000 & 8,000 & .52 & \\
.48 & 8,000 & 7,000 & .53 & \\
.47 & 6,000 & & &
\end{tabular}

In Table 1, $C$ indicates that the corresponding sell order is contingent and the sender of this contingent order wants to buy the other stock at the price in the parenthesis if her contingent claim is successfully executed. Assume that every limit order at each price was given by different investors. Below, we first consider the determination of price and volume under standard call auctions, then under call auction with contingent orders.

\subsubsection{Call Auctions}

By this algorithm price and volume of the opening auction could be confirmed to be: ${ }^{2}$

Stock A: Price-.99 Volume-19,000

Stock B: Price-.50 Volume-12,000

After the opening auction first order book for the continuos auction market will be:

\footnotetext{
${ }^{2}$ To see this, consider Stock A. If $p_{A} \leq 0.97$, since there is no supply, volume is 0 . If $p_{A}=0.98$, then volume is 7,000 (minimum of 7,000 and 37,000 ). If $p_{A}=0.99$, then volume is 19,000 (minimum of 19,000 and 30,000 ). If $p_{A}=1.00$, the volume is 15,000 (minimum of 23,000 and 15,000 ). If $p_{A}=1.01$, then volume is 10,000 (minimum of 29,000 and 10,000 ). If $p_{A} \geq 1.02$, since there is no demand, volume is 0 . For Stock $B$, if $p_{B} \leq 0.49$ or $p_{B} \geq 0.52$, then volume is 0 . If $p_{B}=0.50$, then volume is 12,000 . If $p_{B}=0.51$, then volume 8,000 .
} 
Stock A's Limit Order Book after CA

\begin{tabular}{rr|rr}
\multicolumn{2}{c}{ Buy Orders } & \multicolumn{2}{c}{ Sell Orders } \\
\hline \hline Price & Quantity & Quantity & Price \\
.99 & 11,000 & 4,000 & 1.00 \\
.98 & 7,000 & 6,000 & 1.01 \\
.97 & 8,000 & 8,000 & 1.02 \\
& & 4,000 & 1.03
\end{tabular}

Stock B's Limit Order Book after CA

\begin{tabular}{rr|rr}
\multicolumn{2}{c}{ Buy Orders } & \multicolumn{2}{c}{ Sell Orders } \\
\hline \hline Price & Quantity & Quantity & Price \\
.50 & 6,000 & 9,000 & .51 \\
.49 & 12,000 & 8,000 & .52 \\
.48 & 8,000 & 7,000 & .53 \\
.47 & 6,000 & &
\end{tabular}

Table 2

At the call auction, the investor who is willing to buy Stock B if she can sell Stock A turns out to be a successful seller. Therefore, after the completion of CA, she will be a buyer for Stock B. One can confirm that in the continuous auction trading following opening auction, she will be probably buying from the investor who has contingent interest in Stock A and the latter investor will be buying Stock A. These cross interaction will be adding to the volatility of the early periods of the continuous trading sessions.

\subsubsection{Call Auctions with Contingent Orders}

At the first stage of $\mathrm{CACO}$ algorithm, $p_{1}$ will be identical to resulting CA price.

Stock A: Price-.99 Volume-19,000

Stock B: Price-.50 Volume-12,000

After the first stage algorithm determines the already executed contingent order: the contingent order at Stock A. Hence, the corresponding buy order is entered to the book of A (an order for 22,846 units of stock B with price $.52 \$$ ). In the second stage, the new price vector becomes $p_{2}=(0.99,0.52)$. With this new price vector, algorithm determines a new executed contingent order: contingent order at Stock B. Hence, the corresponding buy order is entered to the book of B (an order for 4,544 units of stock A with price $1.01 \$$ ). At the third stage, the new price vector becomes $p_{3}=(1.00,0.52)$. With completion of the third stage, there are no other contingent orders, so the auction algorithm ends with CACOP equal to $p_{3}$. Resulting limit orders books with the completion of $\mathrm{CACO}$ are given below. 
Stock A's Limit Order Book for CACO

(Last Closing Price is 1.00)

Buy Orders Sell Orders

\begin{tabular}{rr|rr}
\hline \hline Price & Quantity & Quantity & Price \\
\hline 1.01 & 10,000 & 7,000 & .98 \\
1.00 & 5,000 & 12,000 & .99 \\
.99 & 15,000 & 4,000 & 1.00 \\
.98 & 7,000 & 6,000 & 1.01 \\
.97 & 8,000 & 8,000 & 1.02 \\
& & 4,000 & 1.03 \\
\cline { 1 - 1 } 1.01 & 4,544 & &
\end{tabular}

Stock B's Limit Order Book for CACO

(Last Closing Price is .52)

\begin{tabular}{rr|rr}
\multicolumn{2}{c}{ Buy Orders } & \multicolumn{2}{c}{ Sell Orders } \\
\hline \hline Price & Quantity & Quantity & Price \\
\hline .51 & 8,000 & 12,000 & .50 \\
.50 & 6,000 & 9,000 & .51 \\
.49 & 12,000 & 8,000 & .52 \\
.48 & 8,000 & 7,000 & .53 \\
.47 & 6,000 & & \\
& & & \\
.52 & 22, 846 & &
\end{tabular}

Table 3

In Table 3, the italic orders are entered at the consequent stages after the first stage of the algorithm. With this algorithm price and volume of the opening auction will be:

Stock A: Price-1.00 Volume-19,544

Stock B: Price-.52 Volume-22,846

This example illustrates that with CACO, all the information prior to opening is efficiently used by the algorithm and there will be no further trading at the current market structure. Comparing the outcomes of CA and CACO, we also see that CACO trading volume is greater.

Below, we establish that with CACO there are no remaining matching buy and sell orders at the CACOP that can be processed into the continuous trading session. Since this is not the case in CA, we conclude that, in ceteris paribus, early continuous trading session volatility is lower with $\mathrm{CACO}$ than with $\mathrm{CA}$. Moreover, we easily show that trading volume of $\mathrm{CACO}$ is greater than the volume with $\mathrm{CA}$.

Proposition 1 There are no remaining matching buy and sell orders at the CACO. Moreover, the trading volume of $C A C O$ is greater than or equal to the volume with $C A$.

Proof. The first claim follows by definition. Since the algorithm moves to a new stage whenever there are matching buy and sell orders, there cannot be any matching buy and sell limit orders when the algorithm ends. The second claim follows by noting that the volume traded in the first stage of the CACO algorithm is equal to the volume traded in CA. In the following stages (if there are any), the volume traded has to increase. Therefore, the claim follows. 
Remark 1 Since there are no remaining matching buy and sell orders at the CACO, ceteris paribus, the volatility after opening $C A C O$ will be lower than that of opening $C A$.

\section{Incentives Properties of Call Auctions}

In a CA, it is not a weakly dominant strategy to announce the true types (values and quantities). Consider the following example.

Example 2 Consider two buyers with value-quantity pairs $(1,2000),(0.9,1000)$ and two sellers with value quantity pairs $(0.6,2000),(0.5,1000)$; with the last closing/sale price equal to 0.8 . Then all prices in $[0.9,0.6]$ maximize sales (3000 quantities) and 0.8 will be chosen as CAP. However, if buyer 2 and both sellers announce their types thruthfully, buyer 1 has a strict incentive to announce her value as 0.7 , as with that deviation, the price would be 0.7 and she would be strictly better off.

The above example shows that investors may have incentive to misreport their type. However, the incentive to misreport her type arises only when that investor becomes a price setter after the deviation:

Proposition 2 In a CA, given any type announcement of other investors, an investor is never better off (compared to truthful announcement) by announcing another type, unless with that announcement she becomes a price setter (that is, CAP is equal to her announced value).

Proof. Consider a buyer $b_{i}$ whose true value is $v_{i}^{b}$. If by a truthful announcement she is not a successful buyer, then CAP has to be greater than $v_{i}^{b}$. In that case, the only way she can be a successful buyer is to increase her type, which makes the CAP even greater and she obtains a negative utility. Therefore, under this case there is no profitable deviation. If by a truthful announcement she is a successful buyer, then CAP has to be smaller than $v_{i}^{b}$. For a deviation to be profitable, $b_{i}$ has to be a successful buyer after the deviation. If CAP after the deviation is not equal to the new value announcement of $b_{i}$, that means that CAP remained the same. This is because CAP is unaffected by the values announced of the successful buyers, who are not price setters. Therefore, the only way a lie can be beneficial is when after the lie, the investor becomes a price setter (as in the above example). Analogous arguments can be made for the sellers.

A similar version of the above incentive result continues to hold in a CACO.

Proposition 3 In a $C A C O$, given any type announcement of other investors, an investor is never better off (compared to truthful announcement) by announcing another type, unless with the best deviation she becomes a price setter (that is, CACOP is equal to her announced value). 
Proof. For non-contingent investors the arguments are the same as above. Since all the investors care about is the final price that the algorithm produces, for the buyers or the sellers of a stock, the only way to benefit is by becoming a price setter. Now consider a contingent investor who would like to buy stock A only if she can sell stock B. She can lie in two dimensions, value for A and value for B. If she is not a successful seller of Stock B by announcing truthfully-in contrast with CA-she may still want to lie and become a successful seller. This may be the case if her value for Stock A is sufficiently high so that she can offset some loss in selling Stock B by the profit in buying Stock A. Nevertheless, in the best deviation this investor will be becoming a price setter for Stock B. Her incentive in the announcement of values for A is the same as a regular buyer. Hence the result follows.

Propositions 2 and 3 establish that although CA and CACO are not truthful, the incentives from lying could be present only for "price setters." Therefore, we do not expect the behavior of the traders to differ significantly under CA and CACO. Thus, comparing CA and CACO for the same "inputs" is a fair argument, and conclusions of Proposition 1 and Remark 1 are justifiable in this environment.

\section{References}

[1] Economides, N., Schwartz, R., (1995), "Electronic Call Market Trading," Journal of Portfolio Management 21(3), 10-18.

[2] Gatev, E., Goetzmann, W. and Rouwenhorst, K., (2006), "Pairs Trading: Performance of a Relative-Value Arbitrage Rule," Review of Financial Studies, vol. 19(3), 797-827.

[3] Lockwood, L. and Linn, S. (1990), "An Examination of Stock Market Return Volatility During Overnight and Intraday Periods, 1964-1989", The Journal of Finance, Vol. 45, 591-601.

[4] Pagano, M. and Schwartz, R., (2003), "A closing call's impact on market quality at Euronext Paris," Journal of Financial Economics, vol. 68(3), 439-484.

[5] Snell, A. and Tonks I., (2003), "A theoretical analysis of institutional investors' trading costs in auction and dealer markets," Economic Journal, vol. 113(489), 576-597. 